\title{
ATOMIC DATA NEEDED FOR SOLAR ASTRONOMY FROM SPACE
}

\author{
JACQUES DUBAU
}

Observatoire de Paris, 92195 Meudon-Cedex, France

The UV and X-ray wavelength ranges are rich in emission lines from the transition region and the corona, formed at temperatures from $10^{4} \mathrm{~K}$ to $10^{7} \mathrm{~K}$. Fortunately, these emission lines, absorbed by the earth upper atmosphere, can be observed by some solar space observatories. In particular, the NASA SMM satellite, from 1980 to 1990 , has obtained very interesting spectra from which important solar results were derived. In a close future, they will be again observed but with improved spectral, spatial and temporal resolution by SOLAR-A and SOHO.

The japonese SOLAR-A satellite has just been launched (August 1991). Its main purpose is the study of solar active coronal regions, well above $10^{6} \mathrm{~K}$. It is a X-ray instrument which will record continuous and discrete X-ray spectra. For the discrete lines, the BCS instrument, 4 wavelength ranges have been chosen corresponding to $\mathrm{Fe}, \mathrm{Ca}$ and $\mathrm{S} \mathrm{He}$-like ions resonance lines as well as associated satellite lines and $\mathrm{Fe} \mathrm{H}$-like ( $\mathrm{Fe} \mathrm{Ly}_{\alpha}$ ) resonance and satellite lines. From these intense lines, precise temperatures of electron and ions can be derived using simple but realistic coronal plasma models. It is also possible to follow quantitatively the ionization and recombination phase of very hot and transient plasmas, such as flares, and to get some insights to the turbulence and particle acceleration processes. To obtain these informations, many atomic data are inserted in the plasma models. During the eighties, a great experimental and theoretical effort has been done to reach a better understanding of the atomic processes contributing to the intensities of the $\mathrm{H}$-like and He-like resonance and satellite lines and many new atomic parameters have been used in the models to interpret plasma spectra emitted by fusion devices, such as tokamaks, solar and astrophysical plasmas (Bitter et al., 1979,1981; Doschek et al., 1979; Culhane et al., 1981; Bely-Dubau et al., 1979, 1982). In particular, many lines being blended accurate wavelength identifications were required, which could only be obtained from precise experimental measurements. On the other hand, the theoretical importance of recombination and ionization processes on the line intensities has been stressed, this last point being often underestimated but being crucial for transient plasma such as flares.

Other space solar X-ray satellites are planned to be launched in the next future, more precisely the russian CORONAS 2 project for which an X-ray linear polarimeter has been designed. It could measure for $\mathrm{Fe}$ and $\mathrm{Mg} \mathrm{He}$-like ions the linear polarization of the resonance and satellite lines which would be very valuable to detect some particle acceleration processes during solar flare impulsive phase. 
The ESA-NASA SOHO solar observatory contains many UV instruments (CDS, SUMER, UVCS , ..). It will be launched in 1995 during the next sun minimum and has not for purpose to study active solar events but the solar wind and solar oscillations (solar cycle, $\approx 11$ years; last maxima, 1980, 1991). In the UV range, it can observed the photosphere, the chromosphere, the transition region, the inner and outer corona with very high resolutions. The observation of the outer corona, by the UVCS, will be facilitate by the location of the satellite at the Lagrange $L 1$ point $\left(1.5 \times 10^{6} \mathrm{~km}\right)$ to avoid scattered UV solar radiation by the earth atmosphere. As for the former X-ray intruments, many spectral diagnostics will be used. More particularly, with the SUMER and CDS instruments, the shifts of line can give quantitative informations on the direction and velocity of the solar wind and many line ratios are sensitive to the electron density. On the opposite to the $\mathrm{X}$-ray range, very few lines give informations on the electron temperature. Many atomic theoretical works have already been devoted to the ions of interest in this wavelength region and these data are available in the litterature and in the atomic data banks : wavelengths, oscillator strenghs, bound-free photoabsorption cross sections and collision strengths. For most of the usual studies of the quiet solar atmosphere these data will be sufficient but for specific works on the solar wind it is still not clear if other processes will not play an important role. Indeed the solar wind plasma is certainly not in a coronal equilibrium even if it is partly "frozen" : different atomic elements and particles being accelerated differently. There is therefore still some place for new experimental and theoretical atomic works, in particular, very accurate wavelength measurements are important for line shift diagnostics, and theoretical calculations of atomic processes, which would not occured in a coronal equilibrium plasma, could be necessary.

\section{References}

Bitter M., Hill K.W., Sauthoff N.R., Efthimion P.C., Meservey E., Roney W., von Goeler S., Horton R., Goldman M., and Stodiek W.: 1979, Phys. Rev. Lett., 49, 129

Bitter M., von Goeler S., Hill K.W., Horton R., Johnson D., Roney W., Sauthoff N.R., Silver E., and Stodiek W.: 1981, Phys. Rev. Lett., 47, 921

Doschek G.A., Kreplin R.W., and Feldman U.: 1979, Astrophys J., 233, L157

Culhane J.L., Gabriel A.H., Acton L.W., Rapley C.G., Phillips K.J., Wolfson C.J., Antonucci E., Bentley R.D., Catura R.C., Jordan C., Kayat M.A., Kent B.J., Leibacher J.W., Parmar A.N., Sherman J.C., Springer L.A., Strong K.T., and Veck N.J.: 1981, Astrophys J., 244, L141

Bely-Dubau F., Gabriel A.H., and Volonté S.: 1979, Mon. Not. R. astr. Soc., 186, 405

Bely-Dubau F., Dubau J., Faucher P., and Gabriel A.H.: 1982, Mon. Not. R. astr. Soc., 198, 239 